\title{
Visualizing adenosine to inosine RNA editing in single mammalian cells
}

\author{
Ian A. Mellis ${ }^{1,2}$, Rohit Gupte ${ }^{1}$, Arjun Raj ${ }^{1,2,3}$, and Sara H. Rouhanifard ${ }^{1, *}$ \\ ${ }^{1}$ Department of Bioengineering, University of Pennsylvania, Philadelphia PA \\ ${ }^{2}$ Genomics and Computational Biology Group, Perelman School of Medicine, University of \\ Pennsylvania, Philadelphia PA \\ ${ }^{3}$ Department of Genetics, Perelman School of Medicine, University of Pennsylvania, Philadelphia \\ PA
}

\begin{abstract}
Conversion of adenosine to inosine is a frequent type of RNA editing, but important details about its biology remain unknown due to a lack of imaging tools. We developed inoFISH to directly visualize and quantify adenosine-to-inosine edited transcripts in situ. We found that editing of GRIA2, EIF2AK2, and NUP43 is uncorrelated with nuclear localization and paraspeckle association. Further, NUP43 exhibits constant editing levels between single cells while GRIA2 levels vary.
\end{abstract}

\section{Body}

Many RNA species are modified to contain non-canonical bases, a process known as RNA editing. The most prevalent type is adenosine-to-inosine editing ${ }^{1}$, wherein adenosine deaminases (e.g., ADARs) enzymatically modify an adenosine base to an inosine base (Figure 1a), disruption of which leads to defects in hematopoiesis ${ }^{2}$ and neurological function ${ }^{3}$. It has been speculated that adenosine-to-inosine RNA editing influences subcellular localization patterns like nuclear retention ${ }^{4-7}$, but the lack of visualization tools has left this and other hypotheses untested. We thus developed inosineFISH (inoFISH), a fluorescence in situ hybridization-based method for directly imaging adenosine-to-inosine RNA editing events with single-molecule resolution.

Discriminating edited from unedited RNA via RNA fluorescence in situ hybridization (RNA FISH) is difficult because it relies on the hybridization of oligonucleotide probes to visualize the target of interest ${ }^{8}$. Short oligonucleotides bind nonspecifically while long

\footnotetext{
Users may view, print, copy, and download text and data-mine the content in such documents, for the purposes of academic research, subject always to the full Conditions of use: http://www.nature.com/authors/editorial_policies/license.html\#terms

*To whom correspondence should be addressed: sara.rouhanifard@gmail.com. Author Contributions:

SHR, IAM and AR conceived of the paper, SHR and IAM performed experiments, IAM, RG, and AR wrote custom software, IAM, SHR and AR analyzed the data, IAM, SHR and AR wrote the paper.

Competing financial interests:

AR receives royalties and consulting income from Biosearch Technologies related to Stellaris RNA FISH.
} 
oligonucleotides cannot discriminate single-base differences. We thus used a 'toehold probe' strategy ${ }^{9}$ to reduce the initial hybridization region of our detection probes in order to confer selectivity based on single-nucleotide differences (Figure 1b, Supplementary Fig. 1). Our scheme took advantage of the fact that inosine preferentially binds to cytosine rather than thymine by using two detection probes that compete to target the unedited, adenosinebearing sequence using a thymine, and the edited, inosine-bearing sequence using a cytosine. Upon specific binding, the "mask" sequence is released by strand displacement to stabilize hybridization. However, single oligonucleotides are still prone to nonspecific binding, so we simultaneously used smFISH (the "mRNA guide" probe) to target a constant region of mRNA, coupled to a unique fluorophore (Figure 1b). The mRNA guide showed us where to look for specific detection probes.

To test whether inoFISH could visualize adenosine-to-inosine editing, we chose the canonical, well-studied example of the Glutamate receptor 2 transcript (GRIA2). (GRIA2 editing is critical for neuronal function ${ }^{10}$ and defects in GRIA2 editing have been associated with $\mathrm{ALS}^{11}$.) We confirmed that GRIA2 was edited by comparing genomic DNA and cDNA sequence in SH-SY5Y cells (Supplementary Fig. 2), and verified that it is a viable target for smFISH (Supplementary Fig. 3). Combining four biological replicates, $10.53 \%$ of mRNA guides uniquely colocalized with adenosine or inosine detection probes, with $5.25 \%$ and $5.28 \%$ of GRIA2 guides colocalizing with the adenosine-detection and inosine-detection probes respectively Figure 1c, d). The estimated mean editing level for GRIA2 was 57.3\% (95\% confidence interval: $45.1 \%, 69.5 \%$, full statistical model in Supplementary Note).

To confirm that detection probes did not colocalize with guide probes by random chance, we measured the rate of random colocalization by computationally shifting guide spots by 5 pixels in both the $\mathrm{X}$ and $\mathrm{Y}$ direction ("Pixel-shift"), thereby moving them outside the range of any true colocalization events (see Methods; Figure 1d, Supplementary Fig. 4). Pixelshift analysis reduced colocalization to $1.83 \%$ and $1.16 \%$ for adenosine and inosine, respectively, showing that most of colocalization events were specific. (Substituting an unrelated guide probe yielded similar results; Supplementary Fig. 5.) To check for dyespecific effects, we swapped fluorophores on the detection probes (Figure 1d,

Supplementary Fig. 4), revealing variation in the estimated mean editing level of $22 \%$ (Figure 1c, d, Supplementary Figures 6 and 7, and Supplementary Note). Together, these findings show that inoFISH can measure editing levels provided that one checks for dyespecific biases in detection probe sets.

We validated inoFISH estimates of editing levels by comparing them to three established population-based methods (Supplementary Fig. 8). We generated GRIA2 cDNA and estimated editing ratio either through Sanger sequencing or by digesting with a restriction enzyme specific to cDNA from the edited transcript ${ }^{12}$. We also cloned and sequenced individual GRIA2 cDNA molecules. We found editing ratios of 59\%, 54.9\% and 50\%, respectively, consistent with the $57.3 \%$ mean estimated editing level (95\% confidence interval: $[45.1 \%, 69.5 \%]$ ) measured by inoFISH. Publicly available RNA-sequencing data from untreated SH-SY5Y cells also revealed GRIA2 editing (see Methods and Supplementary Fig. 8). 
To verify that inoFISH signals were specific to inosine bases and not adenosine or guanosine bases, we altered the frequency of inosines in two different ways. GRIA2 mRNA is primarily edited by the enzyme ADAR2, so we used siRNA to knock down ADAR2 mRNA levels by $60 \%$ in SH-SY5Y cells ${ }^{13,14}$. We observed a concomitant reduction in mean estimated GRIA2 editing level from 65\% to $14 \%$ (Parametric bootstrap p $=0.0004$, see Supplementary Note; Figure 1e, Supplementary Fig. 6). We also chemically modified inosine bases with acrylonitrile on the $\mathrm{N}^{1}$ position to prevent base pairing to cytosine $\mathrm{e}^{15,16}$, reducing observed editing level from 52.1\% to $13.5 \%$ (Parametric bootstrap $\mathrm{p}=0.0006$ ) (Figure 1f, Supplementary Fig. 6 and 7).

Additionally, we designed a guanosine-carrying "false detection" probe, which should not bind to either the edited or unedited transcript; it did not bind more than expected by chance (Supplementary Fig. 9). These results show that inoFISH specifically discriminates adenosine and inosine bases.

We next measured the subcellular localization of edited and unedited transcripts. Previous studies used cell fractionation to show that unmodified RNAs exist in both the nucleus and the cytoplasm, whereas hyper-edited RNAs—but not selectively edited RNAs—-were predominantly nuclear ${ }^{4,6}$. Other studies have shown that mRNAs containing Alu repeats, which are prone to adenosine-to-inosine editing, are inefficiently exported to the cytoplasm ${ }^{5}$.

We therefore looked for associations between editing status and subcellular localization of GRIA2 transcripts. We classified GRIA2 transcripts as nuclear if they overlapped with the nuclear stain DAPI. Estimated GRIA2 editing levels were roughly equal in both cellular compartments ( $\mathrm{p}=0.38$; Figure 2a, Supplementary Fig. 10). (Uncharacteristically of most mRNAs, $93.4 \%$ of GRIA2 transcripts localized to the nucleus, though they were still translated; Supplementary Fig. 3.)

We also used inoFISH to visualize adenosine-to-inosine editing in two additional targets: the hyper-edited transcript EIF2AK2 $2^{17}$ (Figure 2b) as well as the Alu-bearing NUP43 ${ }^{5}$ (Figure 2c) in U-87 MG cells (Supplementary Fig. 11), which we validated as editing targets by Sanger sequencing of genomic DNA and cDNA (Supplementary Fig. 2). We found that $6.91 \%$ and $5.57 \%$ of EIF2AK2 guide spots colocalized with adenosine- and inosine-specific detection spots, respectively (Figure 2b, Supplementary Fig. 4), giving a population-wide mean editing level estimate of $36.4 \%$ (95\% confidence interval: [20.4\%, 53.1\%]). For NUP43, $11.3 \%$ and $12.4 \%$ of guide spots colocalized with the adenosine- and inosinespecific detection spots (Figure 2c, Supplementary Fig. 4), giving a population-wide editing level estimate of $53.2 \%$ (95\% confidence interval: [ $45.1 \%, 61.2 \%]$ ). In both cases, the editing level did not vary between nucleus and cytoplasm ( $\mathrm{p}=0.18,0.81$ for EIF2AK2, NUP43, respectively); (Figure 2b,c, Supplementary Fig. 10). Note that the three inoFISH targets studied had detection efficiencies of $10 \%, 12 \%$ and $24 \%$; the reasons for this variability is unknown, but it is within previous bounds 9,18 . As before, cyanoethylation reduced the percentage of inosine-detection probe colocalization with guide probe for both EIF2AK2 and NUP43, again showing specificity (Supplementary Fig. 7). 
InoFISH also allowed us to test whether edited transcripts are trafficked to nuclear paraspeckles ${ }^{7}$. We performed inoFISH together with single-molecule RNA FISH of NEAT1 RNA, a marker of nuclear paraspeckles ${ }^{19}$, in SH-SY5Y cells (Figure 2d), revealing that $8.57 \%$ of all GRIA2 transcripts colocalized with paraspeckles (Figure 2d). Simulations (see Methods) showed that the observed rate of GRIA2-paraspeckle association was 1.7-fold greater than expected by random chance (Simulation of GRIA2-paraspeckle association rate null distribution for one representative replicate $\mathrm{p}<0.001$, see Online Methods).

We then used inoFISH to determine whether edited or unedited GRIA2 transcripts were preferentially associated with paraspeckles. We found no significant differences in the editing status in paraspeckles for GRIA2 in SH-SY5Y cells ( $\mathrm{p}=0.44$, determined via simulation, for one representative replicate; Figure 2d), demonstrating that edited GRIA2 transcripts in SH-SY5Y cells do not necessarily preferentially associate with paraspeckles.

We next used inoFISH to determine whether adenosine-to-inosine editing is cotranscriptional or post-transcriptional. Introns mark transcription sites, and colocalization of edited transcripts with intron signal would suggest that editing can occur cotranscriptionally. We concurrently performed NUP43 inoFISH with single-molecule FISH targeting NUP43 introns in 212 U-87 MG cells (Figure 2e), observing 17 total transcription sites, of which 5 transcription sites contained unedited NUP43 and none containing edited NUP43 (Figure 2e). This result does not rule out co-transcriptional editing of NUP43 altogether, but does suggest that some NUP43 editing may be post-transcriptional.

We also looked for evidence of fluctuations in editing level from cell to cell. We simulated inoFISH results in the cases of uniform (Figure 3a) or variable editing levels in single cells (Figure 3b). We found that GRIA2 editing in single cells was not consistent with the constant editing level model, suggesting per-cell heterogeneity in GRIA2 editing levels (Figure 3c). NUP43 editing in U-87 MG cells, however, was consistent with the constantediting level model (Figure 3d). Thus single-cell fluctuations in the level of editing may occur in a target-specific manner.

InoFISH provides a direct method for visualizing adenosine-to-inosine RNA editing in single cells with single-nucleotide resolution. Cell population-wide studies lack the resolution to provide information such as subcellular localization and cell-to-cell variability of RNA editing. This new tool will enable researchers to answer basic questions about edited RNA species and will enable a deeper understanding of the biology of adenosine-to-inosine editing.

\section{Online Methods InoFISH protocol}

The step-by-step inoFISH protocol (description below) can be found in the Supplementary Protocol. 


\section{Cell culture}

We grew human neuroblastoma cells (SH-SY5Y, ATCC CRL- 2266) in a 1:1 mixture of Eagle's Minimum Essential Medium and F12 Medium supplemented with 10\% FBS and 50 $\mathrm{U} / \mathrm{mL}$ penicillin and streptomycin. We grew human glioblastoma cells (U-87 MG, ATCC HTB-14) in Eagle's Minimum Essential Medium supplemented with 10\% FBS and 50 $\mathrm{U} / \mathrm{mL}$ penicillin and streptomycin. Note: Some SH-SY5Y and U87 cells can be autofluorescent when grown on glass slides, this is improved when the cells are $\sim 70 \%$ confluent.

\section{Selection of targets for inoFISH}

Besides the well-studied editing target GRIA2, we wanted to test inoFISH on editing targets that are less commonly studied in the literature. For these we referred to the literature, to the RADAR database of RNA editing, and to publicly available RNA-seq data for screening. We identified NUP43 and EIF2AK2 as targets that are studied in a relatively small number of research groups, that are candidate editing targets in published transcriptome-wide adenosine-to-inosine editing screens, and that have editing sites amenable to inoFISH probe designs. In order to systematically identify inoFISH targets, we found it useful to first filter targets by those with enough non-repetitive sequence to design a full guide probe ( $>2420$ mer oligonucleotides). Then, we performed transcriptome-wide screens aimed at studying editing targets in the RADAR database that are conserved across humans, non-human primates, and mice (http://rnaedit.com/. Accessed: 28th October 2016). We expect that conserved targets are more likely both to be biologically interesting and to be observable in our cell lines than targets that only appear in screening experiments in humans alone.

RNA-sequencing-based screen for candidate editing targets-We downloaded publicly available RNA-sequencing data from EBI ArrayExpress for total RNA from two biological replicates of SH-SY5Y cells ${ }^{20}$ and three replicates of U-87 MG cells ${ }^{21}$. We also downloaded RNA editing candidates' positions (in human genome build hg19) from the RADAR database, with a focus on conserved positions and those derived from neural lineage samples. We aligned raw reads to hg19 with STAR v2.3.0e with default parameters except for runThreadN set to 4. Next, we used picard tools v1.96 (http:// broadinstitute.github.io/picard/. Accessed: 28th October 2016) to interconvert between aligned SAM and BAM file formats (SamFormatConverter), to sort BAMs by coordinate (SortSam), to index sorted BAMs (BuildBamIndex), and to remove PCR duplicates (MarkDuplicates). We then trimmed $5^{\prime}$ ends of all aligned and PCR-deduplicated reads with bamUtil v1.0.13 (http://genome.sph.umich.edu/wiki/BamUtil. Accessed: 28th October 2016). Finally, using bam-readcount $\mathrm{v}$ we filtered aligned reads by those overlapping candidate editing sites of interest, by their overall mapping (phred) score and individual base call qualities using a custom bed file and parameters -q 25 and -b 20. We quantified RNAseq based editing level estimates in $R$ by calculating the fraction of filtered reads with ' $G$ ' base calls at each editing site. We considered for downstream RNA editing verification and inoFISH probe design any sites in these screens with at least seven overlapping filtered reads, of which at least one was called 'G' at the editing site. 
Verification of RNA editing of candidate targets-As described below, we used RTPCR of total RNA and PCR of genomic DNA in cell lines of interest to further check that candidate editing sites were in fact RNA edited in our cell lines.

Optimization of inoFISH targets-We then chose targets for inoFISH by checking RTPCR and genomic DNA PCR Sanger sequencing results for each candidate site to ensure that there would be no additional polymorphisms in transcripts, resulting either from RNA editing or single-nucleotide polymorphisms, in the regions flanking the editing site up to 30bp up- or downstream. (Supplementary Fig. 4). We ultimately designed inoFISH probe sets against one editing site in NUP43 and two editing sites in EIF2AK2 (both using the same guide probe set) that appeared to be amenable to inoFISH guide and detection probe set design. We were able to verify inoFISH probe binding with detection efficiencies greater than expected by random colocalization for the one NUP43 candidate editing site and one of the two EIF2AK2 editing sites. (See below for experimental methods.)

\section{Genotyping of edited regions}

We extracted genomic DNA from SH-SY5Y cells and U-87 MG cells using the Qiagen DNeasy Blood \& Tissue kit. We used Platinum Taq (Invitrogen cat. \#10966-018) for PCR amplification of the genomic regions of interest for each target, following manufacturer's recommendations for reaction component concentrations. We PCR-amplified two biological replicates, each with two technical PCR replicates. For GRIA2, we used primers GRIA2-F1 and GRIA2-R2 (Supplementary table 1). For EIF2AK2, we used EIF2AK2_20-F1 and EIF2AK2_20-R1, and for NUP43 we used NUP43-F1 and NUP43-R1 (Supplementary table 1). We confirmed PCR product sizes by gel electrophoresis, using a $1.5 \%$ agarose gel in TAE. Then, we treated these PCR products with ExoSAP-IT (Affymetrix 78200) according to manufacturer's instructions, and submitted them for Sanger sequencing at the University of Pennsylvania DNA Sequencing facility.

\section{Estimation of editing efficiency by RT-PCR and Sanger sequencing}

We extracted total RNA from SH-SY5Y and U-87 MG cells using miRNeasy kits (Qiagen 217004) according to manufacturer's instructions. Then, we reverse-transcribed target transcripts around editing sites of interest using Superscript III First strand RT kit (ThermoFisher 18080044) according to manufacturer's instructions. In separate reactions for RNA from each cell type, we used both oligo-dT and transcript-specific primers for reversetranscription. Briefly, we used $50 \mathrm{ng}$ of RNA per reaction for reverse transcribed with either oligo-dT or transcript-specific primers (Supplementary table 1). Then, we performed PCR with transcript-specific primers (Supplementary table 1) using Platinum Taq (Invitrogen cat. \#10966-018) according to manufacturer's instructions. We completed biological replicates, each with technical PCR replicates for these reactions. We confirmed product sizes by gel electrophoresis on $1.5 \%$ agarose gels in TAE. Then, we treated these products with ExoSAPIT (Affymetrix 78200) according to manufacturer's instructions and submitted for sequencing at the University of Pennsylvania DNA Sequencing facility. 


\section{Estimation of editing efficiency by clonal analysis of GRIA2 RT-PCR product}

The amplified GRIA2 cDNA was cloned into a vector using the TOPO TA cloning kit (Thermo), transformed into chemically competent Escherichia coli cells, and plated on LB plates with $0.1 \mathrm{mg} / \mathrm{mL}$ ampicillin. We isolated DNA from $>20$ Individual colonies and submitted it for sequencing at the University of Pennsylvania DNA Sequencing Facility. We performed sequence alignment at the editing site using MAFFT in Benchling to determine the ratio of edited and unedited transcripts.

\section{Estimation of editing efficiency by restriction digest and bioanalyzer analysis}

Edited and unedited GRIA2 cDNAs yield distinct restriction fragment patterns upon digestion with $B b V^{22}$. Edited GRIA2 cDNA yields two DNA fragments upon digestion (225 bp and $46 \mathrm{bp}$ ), and unedited GRIA2 cDNA yields three DNA fragments (145 bp, $80 \mathrm{bp}$, and $46 \mathrm{bp}$ ). Following BbvI digestion (NEB R0173S) of GRIA2 cDNA, according to manufacturer's instructions, we submitted digestion products for fragment sizing analysis on an Agilent 2100 Bioanalyzer at the University of Pennsylvania DNA Sequencing Facility.

\section{RNA probe design and synthesis}

For each of the validated editing sites, we designed probes by matching free energies of hybridization as specified in Levesque et al. (2013b). We optimized mask oligonucleotides to leave 8-base-pair (bp) overhangs for each of the detection probes and pooled all five together to act as the complete allele-specific probe. We provide all oligonucleotide sequences in Supplementary Table 1. We coupled 3' amine-labeled adenosine- and inosinedetection probes to NHS-Cy3 or NHS-Cy5 fluorophores (GE Healthcare) and purchased respective guide probes labeled with Cal fluor 610 (Biosearch Technologies). We coupled probes targeting ADAR1, ADAR2 and NEAT1 mRNA to NHS-Atto700. We purified dyecoupled probes by high-performance liquid chromatography.

\section{inoFISH procedure}

We grew cells on glass coverslides until $\sim 70 \%$ confluent. We washed the cells twice with $1 \mathrm{X}$ PBS, then fixed for 10 minutes with $4 \%$ formaldehyde/1X PBS at room temperature. We aspirated off the formaldehyde, and rinsed twice with 1X PBS prior to adding 70\% ethanol for storage at $4^{\circ} \mathrm{C}$ or inoFISH after a one hour permeabilization in $70 \%$ ethanol. We incubated our cells overnight at $37^{\circ} \mathrm{C}$ in hybridization buffer (10\% dextran sulfate, $2 \times \mathrm{SSC}$, $10 \%$ formamide) with $100 \mathrm{nM}$ concentration of guide probe, $24 \mathrm{nM}$ concentration of the adenosine- and inosine-detection probes and $72 \mathrm{nM}$ concentration of the mask probe, ensuring excess mask for complete hybridization to the detection probes. The following morning, we performed two washes in wash buffer (2X SSC, $10 \%$ formamide), each consisting of a $30-\mathrm{min}$ incubation at $37^{\circ} \mathrm{C}$. After the second wash, we rinsed once with $2 \mathrm{X}$ SCC/DAPI and once with anti-fade buffer (10 mM Tris (pH 8.0), $2 X$ SSC, $1 \% \mathrm{w} / \mathrm{v}$ glucose). Finally, we mounted the sample for imaging in an anti-fade buffer with catalase and glucose oxidase (Raj et al. 2008) to prevent photobleaching. We performed RNA FISH on cell culture samples grown on a Lab-Tek chambered coverglass using $50 \mu \mathrm{L}$ of hybridization solution spread into a thin layer with a coverslip and placed in a parafilm-covered culture dish with a moistened Kimwipe to prevent excessive evaporation. 


\section{Imaging}

We imaged each samples on a Nikon Ti-E inverted fluorescence microscope using a 100x Plan-Apo objective (numerical aperture of 1.40) and a cooled CCD camera (Andor iKon 934). For $100 \times$ imaging, we acquired z-stacks ( $0.3 \mu \mathrm{m}$ spacing between stacks) of stained cells in five different fluorescence channels using filter sets for DAPI, Cy3, Calfluor 610, Cy5, and Atto 700. The filter sets we used were 31000v2 (Chroma), 41028 (Chroma), SP102v1 (Chroma), 17 SP104v2 (Chroma) and SP105 (Chroma) for DAPI, Atto 488, Cy3, Atto 647N/Cy5 and Atto 700, respectively. A custom filter set was used for Alexa 594/ CalFluor610 (Omega). We tuned the exposure times depending on the dyes used: 4 seconds for each guide probe, $4000 \mathrm{msec}$ for each of the detection probes, $5000 \mathrm{msec}$ for the NEAT1 probe, and $7000 \mathrm{msec}$ for ADAR1 and ADAR2 probes. We also acquired images in the Atto 488 channel with a $1000 \mathrm{msec}$ exposure as a marker of autofluorescence.

\section{Image analysis}

We first segmented and thresholded images using a custom Matlab software suite (downloadable at https://bitbucket.org/arjunrajlaboratory/rajlabimagetools/wiki/Home). Segmentation of cells was done manually by drawing a boundary around non-overlapping cells. The software then fits each spot to a two-dimensional Gaussian profile specifically on the Z-plane on which it occurs in order to ascertain subpixel-resolution spot locations. Colocalization took place in two stages: In the first stage, guide spots searched for the nearest-neighbor detection probes within a 2.5-pixel (360-nm) window. We ascertained the median displacement vector field for each match and subsequently used it to correct for chromatic aberrations. After this correction, we used a more stringent 1.5-pixel (195-nm) radius to make the final determination of colocalization. In order to test random colocalization due to spots occurring randomly by chance, we took our images and shifted the guide channel by adding 5 pixels $(1.3 \mu \mathrm{m})$ to the $\mathrm{X}$ and $\mathrm{Y}$ coordinates and then performing colocalization analysis.

\section{Autofluorescence subtraction}

For U-87 MG cells, we controlled for punctate autofluorescence by imaging with the 41028 (Chroma) filter set, the 'gfp channel', which we have previously found to be sensitive for autofluorescence in this cell line (data not shown). We performed colocalization as previously described between guide spots and any spot-like autofluorescence called in the gfp channel. In R, we excluded spots colocalizing with this autofluorescence from all inoFISH analyses.

\section{Subcellular localization}

Nuclear localization-We extracted a DAPI nuclear mask as previously described ${ }^{8}$. We call a spot as localized to the nucleus if the guide spot $\mathrm{X}$ and $\mathrm{Y}$ coordinates overlap with the 2D nuclear mask.

Localization to transcription sites-We visualized NUP43 introns by probing with intron-specific probes coupled to Atto 700 and imaging with SP105 filter set. We used the 
txnSiteGUI2 interface within rajlabimagetools to manually curate calls of exon-intron spot colocalization.

Localization to paraspeckles-We visualized paraspeckles by probing with NEAT1specific probes coupled to Atto 700 and imaging with SP105 filter set. We used the txnSiteGUI2 interface within rajlabimagetools to manually curate calls of transcriptparaspeckle association.

\section{In situ cyanoethylation}

Cyanoethylation was performed similarly to previous descriptions ${ }^{15,16}$. We aspirated the $70 \%$ ethanol off of the fixed cells and added cyanoethylation solution $(1.1 \mathrm{M}$ triethylammoniumacetate ( $\mathrm{pH} 8.6$ ) resuspended in $100 \%$ ethanol) with or without $1.6 \mathrm{M}$ acrylonitrile at $70{ }^{\circ} \mathrm{C}$ for $15 \mathrm{~min}$. Use large volume to prevent drying from evaporation. Remove from heat after $15 \mathrm{~min}$ (30 min incubation abolishes guide probe signal) and wash twice with wash buffer ( $2 \times \mathrm{SSC}, 10 \%$ formamide) before beginning inoFISH procedure.

\section{Statistical analysis}

Detection efficiency-For each label (edited or unedited) in each experiment we calculated the mean fraction of transcripts colocalized with a spot of that label over all replicates (excluding 3-color spots). For complete details of this analysis, please see the Supplementary Note.

Population-wide editing level estimation by inoFISH-We define the populationwide editing level estimate as the average over all replicates of the inferred fraction of uniquely labelled guide spots labelled as edited. For a complete description of our estimation of population-wide editing level, please see the Supplementary Note.

Paraspeckle-transcript association rates-In MATLAB, we simulated the exact conditional null distribution of paraspeckle-transcript association rates for each experiment under the null hypothesis that a paraspeckle and a nuclear-localized transcript will only colocalize by chance. For each cell in each experiment, we conditioned on (1) the shape and size of that cell's nucleus, (2) the locations of all paraspeckles in that nucleus, and (3) the number of transcripts of interest (GRIA2 or EIF2AK2) retained in the nucleus. In order to efficiently simulate these distributions, rather than using txnSiteGUI2 as above, we generated 2D masks for paraspeckle locations and called paraspeckle-transcript association when a randomly placed transcript spot overlapped with this mask. We selected the mask size as 25 pixels per paraspeckle spot called--roughly the mean paraspeckle size--based on our inspection of paraspeckles while calling spots (as in Image analysis). For each experiment, we simulated draws from the exact conditional null distribution 1000 times. A raw p-value for paraspeckle-transcript association rate is equal to the fraction of simulations with a higher paraspeckle-transcript association rate. We similarly simulated exact conditional null distributions for paraspeckle-edited-transcript and paraspeckle-uneditedtranscript association rates. 
Single-cell editing level distributions-In R, we assessed single-cell spot counts after inoFISH colocalization as reported by rajlabimagetools (https://bitbucket.org/ arjunrajlaboratory/rajlabimagetools/wiki/Home; in Image analysis), as well as after autofluorescence subtraction (for U-87 MG data). We simulated the null distribution of data likelihood under a null model wherein all cells sharing the same effective editing level: for an experiment with overall estimated editing level equal to $p_{e}$ (above), let $n_{e}{ }^{j}$ be the number of edited transcripts detected in cell $\mathrm{j}$ and $\mathrm{n}_{\mathrm{u}}{ }^{\mathrm{j}}$ be the number the number of unedited transcripts detected in cell $\mathrm{j}$. Under the null model, $\mathrm{n}_{\mathrm{e}}{ }^{\mathrm{j}}$ is drawn from a Binomial with $\left(\mathrm{n}_{\mathrm{e}}{ }^{\mathrm{j}}+\right.$ $\mathrm{n}_{\mathrm{u}}{ }^{\mathrm{j}}$ ) draws and probability $p_{e}$. We simulated single-cell label counts for cells by drawing from these conditional null distributions for each cell 100000 times. We then compared the negative log-likelihood of the observed data, combined over all replicates, with the distribution of negative log-likelihoods of each simulation iteration. A p-value of 0.12 indicates that $12 \%$ of the simulated iterations had a negative log-likelihood that was greater than the observed data. Note that the - $\log$ (likelihood) density plots in Fig. 3 are subsampled to 3000 of the aforementioned 100000 such iterations per plot, in order to facilitate figure generation. For complete details of this analysis, please see the Supplementary Note.

\section{siRNA knockdowns of ADAR2}

Briefly, we used Lipofectamine RNAimax to transfect SH-SY5Y cells with Silencer Select siRNAs targeting ADARB1 (ADAR; ID:s1011, Ambion) and a Negative Control siRNA (\#1, Ambion) for $72 \mathrm{hrs,} \mathrm{verifying} \mathrm{knockdown} \mathrm{via} \mathrm{RNA} \mathrm{FISH} \mathrm{of} \mathrm{ADAR2.}$

\section{SFPQ-guided GRIA2 inoFISH}

We performed GRIA2 inoFISH, as described above, but substituted a smFISH probe set for SFPQ ${ }^{23}$ for the GRIA2 guide. Like GRIA2 transcripts, SFPQ transcripts are localized to the nucleus in SH-SY5Y cells. In parallel we performed regular GRIA2 inoFISH on a sample of cells from the same passage. We counted the number of GRIA2 mRNA per cell in the regular sample, and subsampled the SFPQ "guide" spots from that distribution of mRNA counts per cell. In this way, we could more directly compare colocalization rates with GRIA2 detection probes between SFPQ-guided and GRIA2-guided experiments. We then performed colocalization as described above.

\section{Cell cycle inhibitor}

We measured nuclear retention of GRIA2 mRNA by inhibiting transcription for $24 \mathrm{hr}$ by applying aphidicolin at $1 \mathrm{ug} / \mathrm{ml}$.

\section{Code Availability Statement}

Scripts for all analyses presented in this paper, including all data extraction, processing, and graphing steps are freely accessible at the following url: https://www.dropbox.com/sh/ j5umuneita1nck9/AAA4W4I648glUUhePJfXyaRaa?dl=0.

\section{Data Availability Statement}

All imaging and other non-RNA-sequencing data associated with this paper are also freely available at the following url: https://www.dropbox.com/sh/vwwnwrmgg72o75c/ 
AACsFK6VbJHY2S5MK8JLR2JNa?dl=0. For publicly available RNA-seq data discussed in Supp. Fig. 1, please see EBI ArrayExpress (https://www.ebi.ac.uk/arrayexpress/) accession numbers E-MTAB-2690 (SH-SY5Y samples "SY5Y_A" and "SY5Y_B") and EMTAB-1875 (U-87 MG samples "s_2_78", “s_2_82", and “s_2_88”).

\section{Supplementary Material}

Refer to Web version on PubMed Central for supplementary material.

\section{Acknowledgments}

We thank Drs. Nishikura and Sakurai for helpful discussions, as well as Caroline Bartman and Benjamin Emert for comments and suggestions. IAM acknowledges support from NIH/NIGMS T32GM007170 (University of Pennsylvania MSTP), NIH/NHGRI T32HG000046 and NIH/NINDS F30NS100595 and SHR from NIH 1F32GM120929-01A1 and AR from an NSF CAREER Award 1350601, NIH New Innovator 1DP2OD008514, NIH/NIBIB R33 EB019767, and NIH 4DN U01 HL129998, and NIH Center for Photogenomics RM1 HG007743.

\section{References (for main text)}

1. Bass BL, Weintraub H. A developmentally regulated activity that unwinds RNA duplexes. Cell. 1987; 48:607-613. [PubMed: 2434241]

2. Liddicoat BJ, et al. RNA editing by ADAR1 prevents MDA5 sensing of endogenous dsRNA as nonself. Science. 2015; 349:1115-1120. [PubMed: 26275108]

3. Higuchi M, et al. Point mutation in an AMPA receptor gene rescues lethality in mice deficient in the RNA-editing enzyme ADAR2. Nature. 2000; 406:78-81. [PubMed: 10894545]

4. Kumar M, Carmichael GG. Nuclear antisense RNA induces extensive adenosine modifications and nuclear retention of target transcripts. Proc Natl Acad Sci U S A. 1997; 94:3542-3547. [PubMed: 9108012]

5. Chen LL, Carmichael GG. Altered nuclear retention of mRNAs containing inverted repeats in human embryonic stem cells: functional role of a nuclear noncoding RNA. Mol Cell. 2009; 35:467478. [PubMed: 19716791]

6. Zhang Z, Carmichael GG. The fate of dsRNA in the nucleus: a p54(nrb)-containing complex mediates the nuclear retention of promiscuously A-to-I edited RNAs. Cell. 2001; 106:465-475. [PubMed: 11525732]

7. Prasanth KV, et al. Regulating gene expression through RNA nuclear retention. Cell. 2005; 123:249-263. [PubMed: 16239143]

8. Raj A, van den Bogaard P, Rifkin SA, van Oudenaarden A, Tyagi S. Imaging individual mRNA molecules using multiple singly labeled probes. Nat Methods. 2008; 5:877-879. [PubMed: 18806792]

9. Levesque MJ, Ginart P, Wei Y, Raj A. Visualizing SNVs to quantify allele-specific expression in single cells. Nat Methods. 2013; 10:865-867. [PubMed: 23913259]

10. Seeburg PH, Single F, Kuner T, Higuchi M, Sprengel R. Genetic manipulation of key determinants of ion flow in glutamate receptor channels in the mouse. Brain Res. 2001; 907:233-243. [PubMed: 11430906]

11. Yamashita T, et al. RNA editing of the Q/R site of GluA2 in different cultured cell lines that constitutively express different levels of RNA editing enzyme ADAR2. Neurosci Res. 2012; 73:42-48. [PubMed: 22366356]

12. Paschen W, Hedreen JC, Ross CA. RNA editing of the glutamate receptor subunits GluR2 and GluR6 in human brain tissue. J Neurochem. 1994; 63:1596-1602. [PubMed: 7523595]

13. Melcher T, et al. A mammalian RNA editing enzyme. Nature. 1996; 379:460-464. [PubMed: 8559253] 
14. O'Connell MA, Gerber A, Keller W. Purification of human double-stranded RNA-specific editase 1 (hRED1) involved in editing of brain glutamate receptor B pre-mRNA. J Biol Chem. 1997; 272:473-478. [PubMed: 8995285]

15. Yoshida M, Ukita T. Modification of nucleosides and nucleotides. VII Selective cyanoethylation of inosine and pseudouridine in yeast transfer ribonucleic acid. Biochim Biophys Acta. 1968; 157:455-465. [PubMed: 5665898]

16. Sakurai M, Yano T, Kawabata H, Ueda H, Suzuki T. Inosine cyanoethylation identifies A-to-I RNA editing sites in the human transcriptome. Nat Chem Biol. 2010; 6:733-740. [PubMed: 20835228]

17. Wang IX, et al. ADAR regulates RNA editing, transcript stability, and gene expression. Cell Rep. 2013; 5:849-860. [PubMed: 24183664]

18. Shaffer SM, et al. Multiplexed detection of viral infections using rapid in situ RNA analysis on a chip. Lab Chip. 2015; 15:3170-3182. [PubMed: 26113495]

19. Sunwoo H, et al. MEN epsilon/beta nuclear-retained non-coding RNAs are up-regulated upon muscle differentiation and are essential components of paraspeckles. Genome Res. 2009; 19:347359. [PubMed: 19106332]

20. Duffy DJ, et al. Integrative omics reveals MYCN as a global suppressor of cellular signalling and enables network-based therapeutic target discovery in neuroblastoma. Oncotarget. 2015; 6:4318243201. [PubMed: 26673823]

21. Kuhlwilm M, Davierwala A, Pääbo S. Identification of putative target genes of the transcription factor RUNX2. PLoS One. 2013; 8:e83218. [PubMed: 24349465]

22. Whitney NP, et al. Calcium-permeable AMPA receptors containing Q/R-unedited GluR2 direct human neural progenitor cell differentiation to neurons. FASEB J. 2008; 22:2888-2900. [PubMed: 18403631]

23. Cabili MN, et al. Localization and abundance analysis of human lncRNAs at single-cell and singlemolecule resolution. Genome Biol. 2015; 16:20. [PubMed: 25630241] 


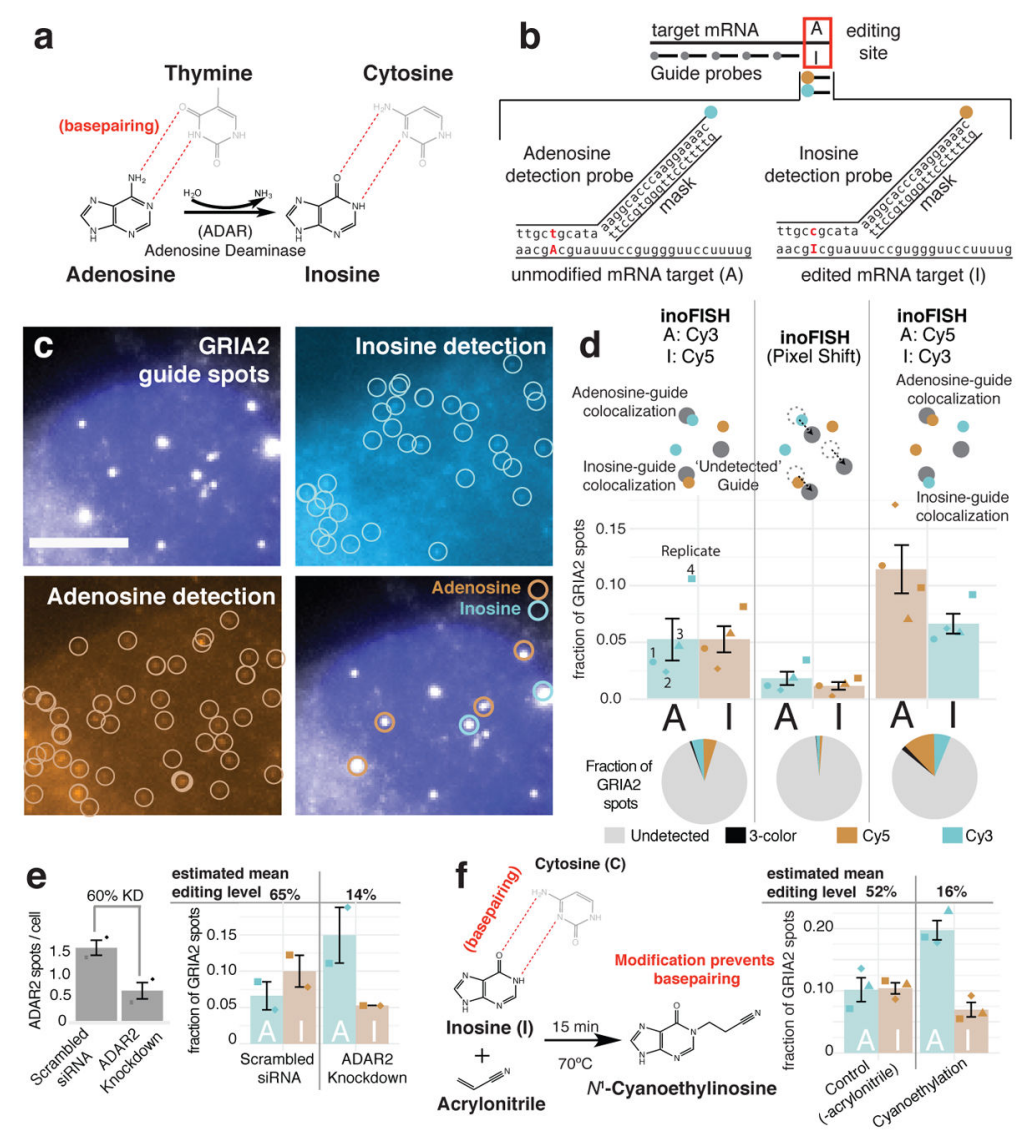

Fig 1. inoFISH discriminates adenosine- and inosine-bearing transcripts in situ (a) Inosine preferentially binds to cytosine rather than thymine. (b) inoFISH probe hybridization scheme. (c) Fluorescence micrographs of Cal Fluor 610-labeled guide probe detecting GRIA2 mRNA (upper left), Cy5-labeled inosine detection probe (upper right) and Cy3-labeled adenosine detection probe (lower left), colocalized (lower-right). Scale bar, 5 $\mu \mathrm{m}$. (d) GRIA2 inoFISH results in SH-SY5Y cells (4 biological replicates), including pixelshift and dye-swap controls; inoFISH probe detection efficiencies per-replicate (points) and mean +/- s.e.m.. Full summary of guide spot labels (pies; mean over all replicates). (e) $A D A R 2$ transcript abundance upon siRNA-mediated ADAR2 knockdown (left); GRIA2 inoFISH results in SH-SY5Y cells $(\mathrm{n}=2)$ after $A D A R 2$ knockdown and scrambled siRNA control (right). (f) Cyanoethylation reaction schematic (left) and GRIA2 inoFISH (right) results in SH-SY5Y cells $(\mathrm{n}=3)$ after in situ cyanoethylation with -acrylonitrile control. 

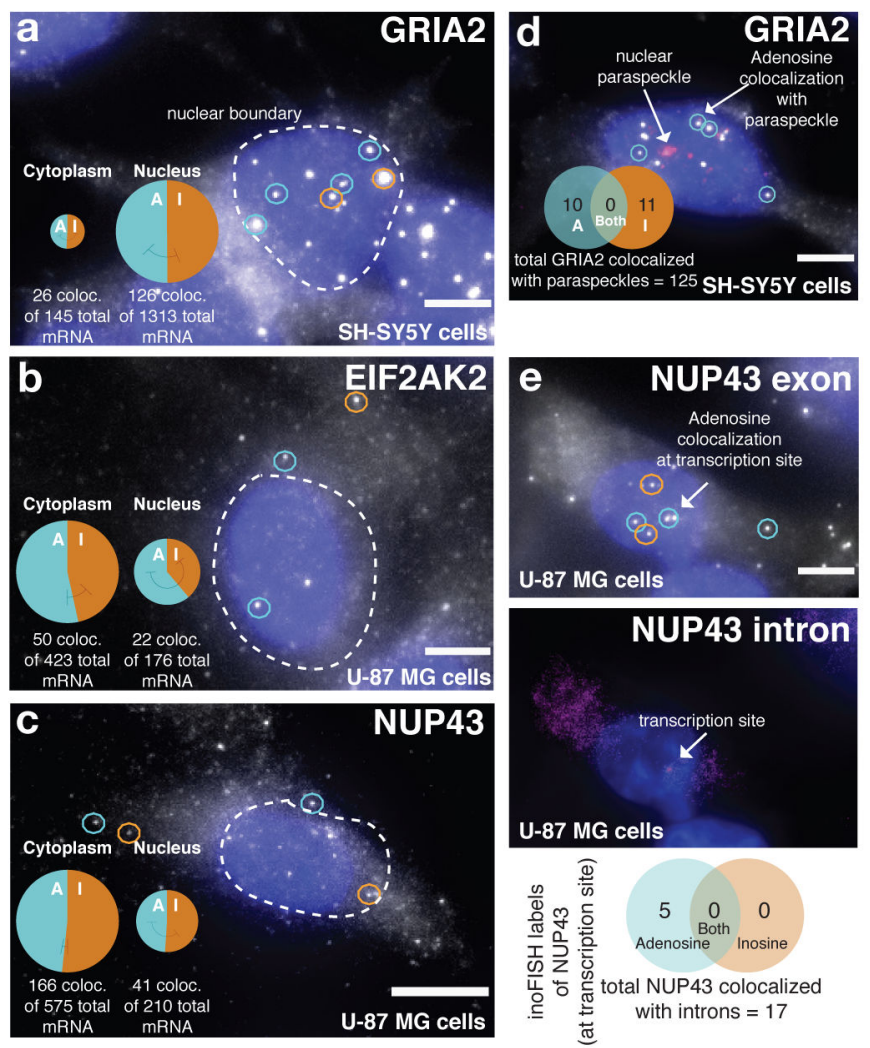

Fig. 2. Analysis of subcellular localization using inoFISH

Nuclear localization analysis reveals no significant differences (parametric bootstrapping) in editing levels for each target between nucleus and cytoplasm. (a) GRIA2 ( $\mathrm{n}=4$ biological replicates; $\mathrm{p}=0.38)$, (b) $E I F 2 A K 2(\mathrm{n}=3 ; \mathrm{p}=0.18)$ and (c) $N U P 43(\mathrm{n}=2 ; \mathrm{p}=0.81)$ transcripts: representative overlays and fractions of labeled transcripts found to be unedited or edited (inlay). (d) GRIA2 inoFISH results with NEAT1-colocalization pooled over $\mathrm{n}=4$ biological replicates: representative overlays and counts of inoFISH-labeled, paraspeckleassociated transcripts. No significant difference between population-wide editing level and paraspeckle-associated editing level (simulation of conditional null per replicate; $\mathrm{p}=0.44$ for one representative replicate) (e) NUP43 inoFISH results with transcription site localization analysis $(n=2)$ : representative images and counts of inoFISH-labeled, transcription site-associated NUP43 transcripts. 


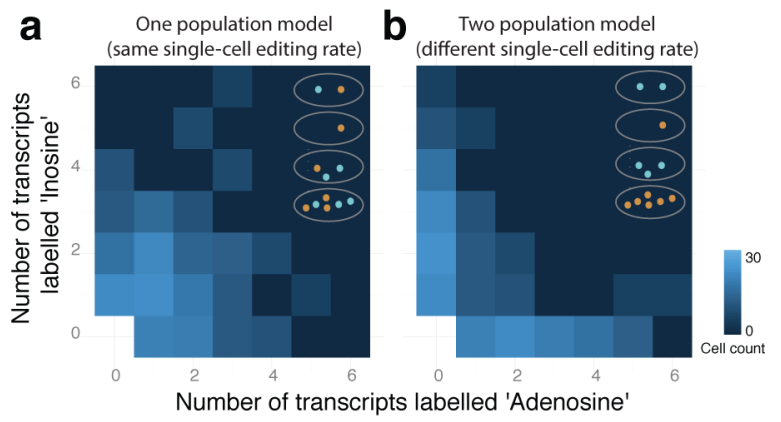

C

Single-cell analysis of GRIA2 inoFISH

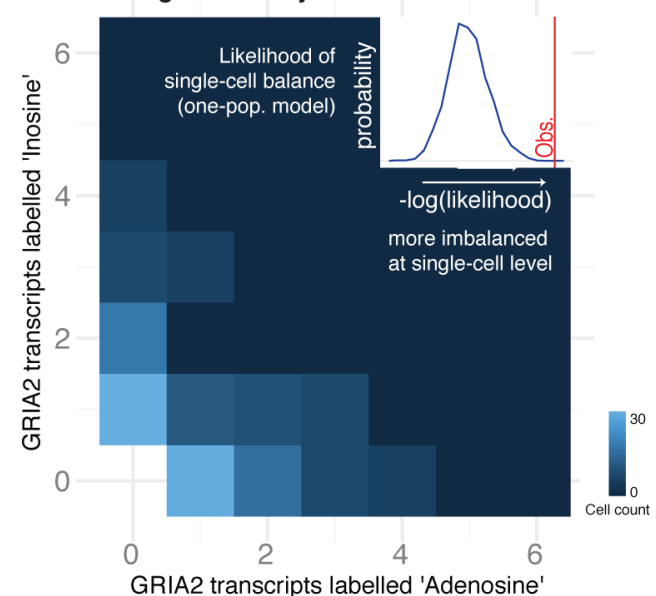

d Single-cell analysis of NUP43 inoFISH

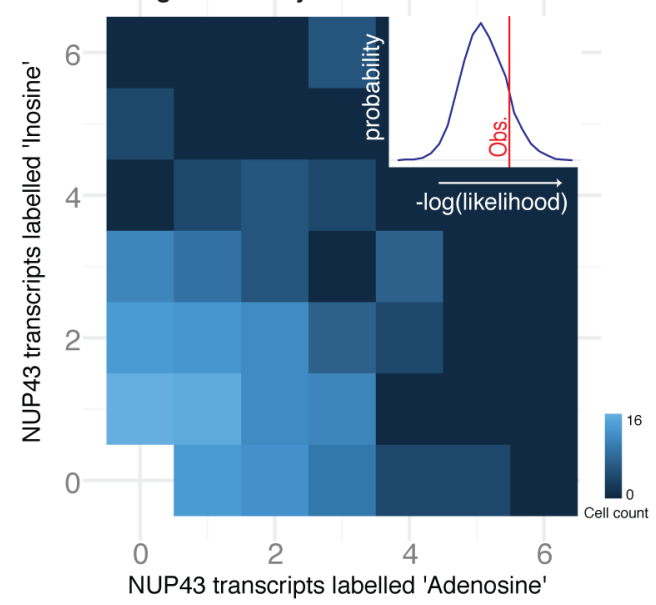

Fig. 3. Single-cell analysis of inoFISH

Simulated inoFISH results assuming (a) binomially-distributed per-cell counts of edited and unedited transcripts; or (b) two populations of cells, one population with 95\% editing and the other with 5\% editing, mixed in proportion according to the population-wide editing level (c) Single-cell analysis of GRIA2 inoFISH results pooled over all 4 replicates and simulation of the exact conditional null distribution of -log(likelihood) of the data under the binomial model specified in (a)(inset) (d) Single-cell analysis of NUP43 inoFISH results pooled over all 4 replicates (left) and simulation of the exact conditional null distribution of - 
$\log$ (likelihood) of the data under the binomial model specified in (a) (inset, see Online Methods). 